\title{
Splitting of high power, cw proton beams
}

\author{
Alberto Facco* and Rita Paparella \\ INFN-Laboratori Nazionali di Legnaro, viale dell'Università, 2, I-35020 Legnaro, Padova, Italy \\ Dan Berkovits \\ SOREQ NRC, Yavne 81800, Israel \\ Isao Yamane \\ KEK, High Energy Accelerator Research Organization, Oho 1-1, Tsukuba, Ibaraki 305, Japan
}

(Received 25 June 2007; published 13 September 2007)

\begin{abstract}
A simple method for splitting a high power, continuous wave (cw) proton beam in two or more branches with low losses has been developed in the framework of the EURISOL (European Isotope Separation OnLine Radioactive Ion Beam Facility) design study. The aim of the system is to deliver up to $4 \mathrm{MW}$ of $\mathrm{H}^{-}$ beam to the main radioactive ion beam production target, and up to $100 \mathrm{~kW}$ of proton beams to three more targets, simultaneously. A three-step method is used, which includes magnetic neutralization of a fraction of the main $\mathrm{H}^{-}$beam, magnetic splitting of $\mathrm{H}^{-}$and $\mathrm{H}^{0}$, and stripping of $\mathrm{H}^{0}$ to $\mathrm{H}^{+}$. The method allows slow raising and individual fine adjustment of the beam intensity in each branch.
\end{abstract}

DOI: 10.1103/PhysRevSTAB.10.091001

PACS numbers: 29.27.Ac, 41.85.Ct, 41.85.Lc

\section{INTRODUCTION}

One of the requirements of the European Isotope Separation On-Line Radioactive Ion Beam Facility (EURISOL) [1] is the possibility of delivering, simultaneously, $1 \mathrm{GeV}$, continuous wave (cw) proton (or $\mathrm{H}^{-}$) beams to a neutron converter (up to $4 \mathrm{MW}$ ), and to one or more direct targets for radioactive ion beam (RIB) production (up to $100 \mathrm{~kW}$ each). Once the main beam is set up in the $4 \mathrm{MW}$ target, the beam on each of the three direct targets must be raised slowly, to avoid dangerous thermal and mechanical stresses, and finely adjusted to the required value for optimum RIB production. This operation must be done without significantly perturbing the main $4 \mathrm{MW}$ beam. The EURISOL driver is designed to accelerate to $1 \mathrm{GeV}$ up to $5 \mathrm{~mA}$ of $\mathrm{H}^{-}$beam (in addition to $100 \mu \mathrm{A}$ of ${ }^{3} \mathrm{He}^{++}$at $2 \mathrm{GeV}$ and $5 \mathrm{~mA}$ of deuterons at $250 \mathrm{MeV}$ ). Another requirement is a very low rate of beam losses, below $1 \mathrm{~W} / \mathrm{m}$ to allow hands-on maintenance [2].

A natural way to split a $\mathrm{H}^{-} \mathrm{cw}$ beam is to strip part of it, in order to change its $\mathrm{A} / \mathrm{q}$ and separate it from the parent beam by means of a dipole magnet. Different techniques have been developed for this aim, mainly combining foil stripping, magnetic stripping, and laser stripping [3-6]. The main problems encountered were beam losses, beam emittance growth, untolerably short foil lifetime, or insufficient laser power. These problems are even more pronounced in the EURISOL case of high power, cw beam, with the requirement of low beam losses for hands-on maintenance. A simple foil stripper leads to three different beams $\left(\mathrm{H}^{-}, \mathrm{H}^{0}, \mathrm{H}^{+}\right)$, and could not hold the $4 \mathrm{MW}$ beam power. Laser stripping is a very successful and promising

\footnotetext{
*facco@lnl.infn.it
}

technique that, in our case, would require high power, $\mathrm{cw}$ lasers, not available at present. We propose a technique that fulfills all EURISOL requirements in a simple and cost effective way, first separating part of the beam by magnetic neutralization, and then transforming it in a proton beam by foil stripping. This method, which uses in a different way elements of the LAMPF PSR injection system [3], can lead to very low losses and long foil lifetime, and can be repeated along the main beam line.

\section{FRACTIONAL NEUTRALIZATION OF $\mathrm{H}^{-}$BEAM BY LORENTZ STRIPPING}

When an $\mathrm{H}^{-}$ion moves in a magnetic field $B$, it experiences a Lorentz force that bends its trajectory and also tends to strip its electrons. For a particle traveling at a speed $\beta$, a transverse magnetic field in the laboratory frame produces a transverse electric field in the rest frame of the particle. According to the Lorentz transformation of the fields,

$$
\left|E_{\perp}\right|=\beta \gamma c\left|B_{\perp}\right| .
$$

This electric field can remove the extra electron of a $\mathrm{H}^{-}$ ion; the $\mathrm{H}^{-}$lifetime, in its rest frame, has been expressed by Scherk [7]:

$$
\begin{aligned}
& \qquad \tau(z)=\left(\frac{A_{1}}{E_{\perp}}\right) \exp \left(\frac{A_{2}}{E_{\perp}}\right), \\
& \text { with }\left\{\begin{array}{l}
A_{1}=2.47 \times 10^{-6} \mathrm{~V} \cdot \mathrm{s} / \mathrm{m} \\
A_{2}=4.49 \times 10^{9} \mathrm{~V} / \mathrm{m} .
\end{array}\right.
\end{aligned}
$$

The fraction $f$ of the stripped ions per unit path length can be expressed as [8]: 


$$
\frac{d f}{d z}=-\frac{f}{(\beta \gamma c \tau)}=\left(\frac{f B}{A_{1}}\right) \exp \left(-\frac{A_{2}}{(\beta \gamma c B)}\right)
$$

For $1 \mathrm{GeV} \mathrm{H}^{-}$ions, a magnetic field of several kilo-gauss is sufficient to strip the first electron, due to its low binding energy $(0.755 \mathrm{eV})$. Magnetic stripping is an ideal tool for neutralizing $\mathrm{H}^{-}$ions, since the $\mathrm{H}^{0}$ production rate can be finely adjusted by varying $B$. Moreover, no $\mathrm{H}^{+}$is produced, due the large binding energy $(13.6 \mathrm{eV})$ of the remaining electron in the $\mathrm{H}^{0}$ ground state. To detach this electron, an extremely high magnetic field (around $40 \mathrm{~T}$ ) would be required; for this reason, to produce $\mathrm{H}^{+}$, either $\mathrm{H}^{0}$ can be excited to a level with low binding energy before passing it through a magnetic stripper [4], or other methods must be used (e.g. a carbon foil stripper).

An unwanted side effect of the magnetic neutralization is emittance growth. The $\mathrm{H}^{-}$ions travel in a curved trajectory in the magnetic field; when they lose their extra electron, the so-generated $\mathrm{H}^{0}$ will approximately keep the instantaneous direction of the tangent of this curve. Particles neutralized at the very beginning will maintain the direction of the incoming $\mathrm{H}^{-}$beam, while particles neutralized at the very end will have the same direction of the one coming out. Since stripping is a probabilistic process, the final $\mathrm{H}^{0}$ atoms will be distributed between these two angles and the beam angular spread will be roughly increased by this amount [9]. Another source of emittance growth is the fact that, due to the curved trajectory in the magnet, the $\mathrm{H}^{0}$ particles are created with transverse coordinates that depend on their position along the curve. The transverse size of the neutral beam is roughly increased by the sagitta of this curve. It is clear that the shorter the magnet, the less emittance growth will be produced by these two effects.

A method to reduce the emittance growth is using a magnetic wiggler. The bending curve is divided in shorter sections, and all angular spreads produced by the different sections are superimposed to each other [9]. Moreover, a wiggler can be designed in order to keep the output $\mathrm{H}^{-}$ beam on the same axis as the input one, no matter what the field strength is; this allows changing the $\mathrm{H}^{0}$ fraction without perturbing the main beam final trajectory.

\section{THE EURISOL BEAM SPLITTING SCHEME}

A three-step splitting scheme was developed, which combines a magnetic neutralizer to extract a $100 \mathrm{~kW} \mathrm{H} \mathrm{H}^{0}$ beam from the original $\sim 4 \mathrm{MW} \mathrm{H}^{-}$one, a bending magnet to separate $\mathrm{H}^{0}$ from $\mathrm{H}^{-}$, and then a stripper foil on the $\mathrm{H}^{0}$ line to strip the neutral beam into a proton beam that can be transported to the targets (see Fig. 1). One more dipole separates the protons from the residual $\mathrm{H}^{0}$ particles that are collected by a beam dump. This scheme can be repeated many times to produce $\mathrm{cw}$ beams in parallel, provided that the main beam is not perturbed by the magnetic neutralizer.

The $\mathrm{H}^{-}$and $\mathrm{H}^{+}$beam dynamics has been calculated with the PARTRAN and TRACEWIN codes [10]. The $\mathrm{H}^{-}$primary beam, with $176 \mathrm{MHz}$ bunch frequency and $5 \mathrm{~mA}$ current, has been simulated using 100000 macroparticles with $3 \times 2 \mathrm{D}$ Gaussian distribution, with normalized $\mathrm{rms}$ emittance $\varepsilon_{x}=\varepsilon_{y}=0.3 \pi \mathrm{mm} \mathrm{mrad}, \quad \varepsilon_{z}=$ $0.4 \pi \mathrm{mm} \mathrm{mrad}$ and $\Delta \mathrm{E}_{\mathrm{rms}}=450 \mathrm{keV}$.

\section{A. Beam line components}

\section{Matching sections}

Four quadrupoles in front of each splitting section are needed to allow beam focusing, as well as a quadrupole doublet after the second bending magnet, in the proton section. The quadrupoles total aperture is $10 \mathrm{~cm}$.

\section{Magnetic neutralizer}

The $\mathrm{H}^{-}$beam is neutralized inside an unconventional wiggler magnet (Fig. 2), which is a chicane consisting of three dipole magnets separated by $40 \mathrm{~mm}$ (required to house the coils), with the same length of $30 \mathrm{~mm}$ but different magnetic field. The necessary gap height is estimated to be $30 \mathrm{~mm}$. The neutralization is concentrated in the center magnet, set at the field strength required to neutralize $2.5 \%$ of the $\mathrm{H}^{-}$beam (EURISOL requirement); this is about $0.66 \mathrm{~T}$ (see graph in Fig. 3), easily reachable with standard technologies. The 1st and 3rd magnets are set at one-half of this value to have nearly no neutralization.

The aim of this system is to have a short stripping path, concentrated in the center magnet, to minimize emittance growth, and, at the same time, an optical system that keeps

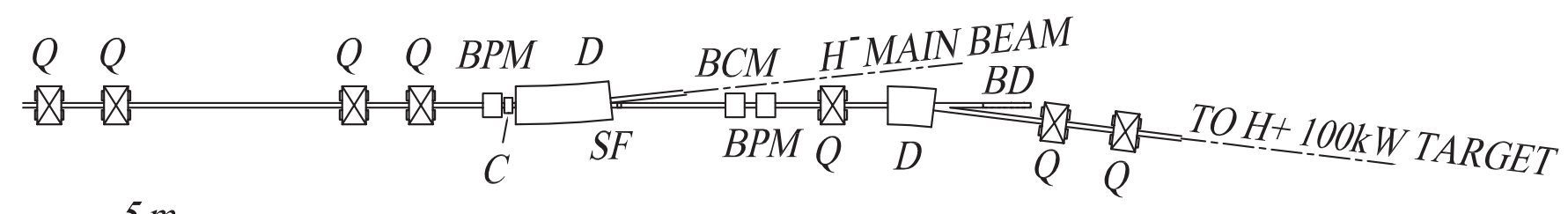

$5 \mathrm{~m}$

FIG. 1. Splitter section based on a wiggler magnet (C). After the first bending magnet (D), $\mathrm{H}^{-}$and $\mathrm{H}^{0}$ beams are separated and sent, respectively, to the second splitter section and to the stripper foil $(\mathrm{SF})$, to be stripped into $\mathrm{H}^{+}$. $\mathrm{Q}=$ quadrupole magnet; $\mathrm{BD}=$ beam dump; $\mathrm{BCM}=$ beam current monitor; $\mathrm{BPM}=$ beam profile monitor. 


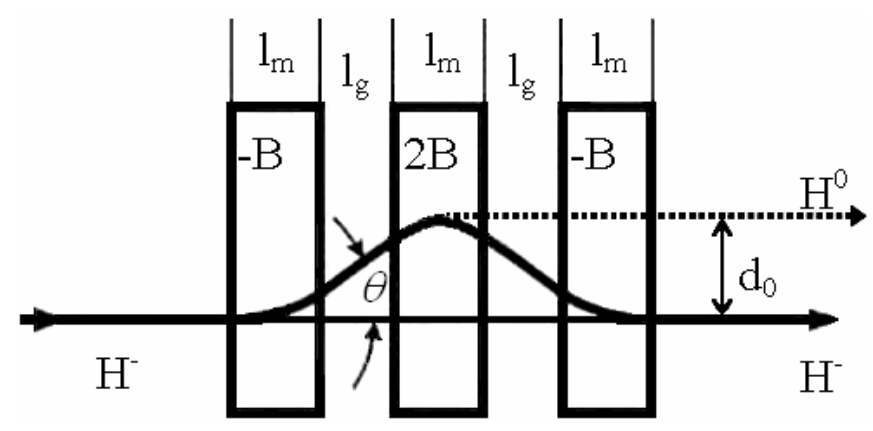

FIG. 2. Schematic representation of the wiggler magnet. $l_{m}$ is the magnet length, $l_{g}$ the gap between magnets, $B$ is the magnetic field, $\theta$ is the deflection angle in the first and third magnets of the wiggler, and $d_{0}$ is the final displacement of the $\mathrm{H}^{0}$ beam from the $\mathrm{H}^{-}$one.

the output $\mathrm{H}^{-}$beam unchanged when the magnetic field $B$ is varied.

The chicane beam optics is rather insensitive to the dipole fringe fields, since these short rectangular magnets with a small bending angle $\left(\theta \sim 0.1^{\circ}\right.$ at the operation fields) produce a very weak focusing in both the horizontal and vertical planes [11]. The fringe fields have been calculated, for different pole shapes, with the standard 1st and 2nd order approximations [12] used in TRACEWIN and in many other codes. The $\mathrm{H}^{-}$beam transport has been simulated with square-edged and Rogowski dipole geometries, as well as with linear fringe field and with no fringe field at all, obtaining always the same results, as expected. A complete field map simulation is anyhow planned as a future development.

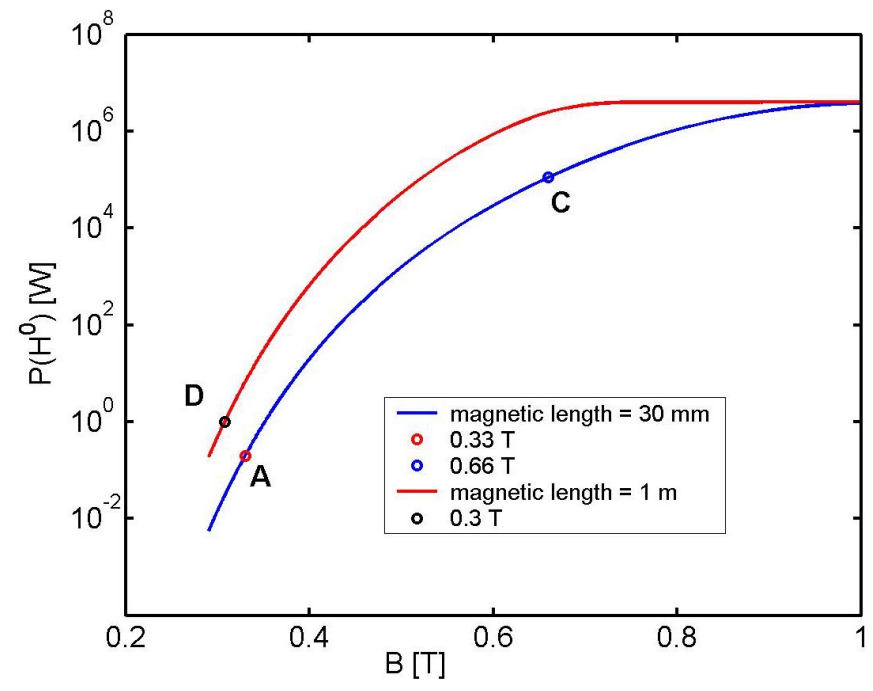

FIG. 3. (Color) Neutralization rate vs $B$ for $1 \mathrm{GeV}, 4 \mathrm{MW} \mathrm{H}^{-}$ beam in a $30 \mathrm{~mm}$ (blue line) and in a $1 \mathrm{~m}$ (red line) dipole magnet. The circles represent the working points of: (A) 1st and 3rd chicane dipoles $\left(0.33 \mathrm{~T}, \mathrm{H}^{0}\right.$ production $\left.<0.1 \mathrm{~W}\right)$; (C) center chicane dipole $\left(0.66 \mathrm{~T}, \mathrm{H}^{0}\right.$ production $\left.100 \mathrm{~kW}\right)$; (D) bending dipole $\left(0.3 \mathrm{~T}, \mathrm{H}^{0}\right.$ production $\left.1 \mathrm{~W} / \mathrm{m}\right)$.
Although the total power of the electrons created at the 2nd magnet, and strongly bent by the downstream magnetic fields, is only about $50 \mathrm{~W}$, a cooled collector should be foreseen at the beam chamber walls.

\section{3. $\mathbf{H}^{0}$ beam emittance}

The important parameter in the chicane design is the emittance of the generated $\mathrm{H}^{0}$ beam. The emittance growth of the parent $\mathrm{H}^{-}$beam was found to be negligible. The $\mathrm{H}^{0}$ beam is produced with a $2 \theta$ angular spread [ $\sin \theta=$ $\left.B l_{m} /(B \rho)\right]$ and with a transverse displacement of its axis of $d_{0} \sim\left(l_{g}+l_{m}\right) \theta$. In Fig. 4 the transverse phase spaces of the $\mathrm{H}^{-}$and $\mathrm{H}^{0}$ beams are shown. The generated $\mathrm{H}^{0}$ phase space distribution was calculated starting from the trajectories of the parent $\mathrm{H}^{-}$particles, assumed with a Gaussian distribution in the phase space.

The total horizontal half angular spread of the $\mathrm{H}^{-}$beam, at the chicane input, is about $1.6 \mathrm{mrad}$ [Fig. 4(a)].

At the chicane output, the centroid of the generated $\mathrm{H}^{0}$ beam is displaced in $x$ by $0.11 \mathrm{~mm}$, and its horizontal half angular spread is about $3.3 \mathrm{mrad}$ [Fig. 4(c)]. As a consequence, the $\mathrm{H}^{0}$ horizontal emittance is increased to $\varepsilon_{x}=$ $0.78 \pi \mathrm{mm} \mathrm{mrad}$, i.e., about $260 \%$ of the parent beam one; this is still an acceptable value for transporting the beam to the RIB targets without beam losses.

In the $\mathrm{H}^{0}$ distribution calculation, in order to keep the results independent from the particular choice of fringe field, the rather general approximation of constant magnetic field along the all center magnet was used. This is a conservative assumption; for any pole shape, in fact, the rather low aspect ratio (length/gap height) of the dipole gives a realistic magnetic field distribution which is peaked at its center, thus concentrating around this point the $\mathrm{H}^{0}$ production and shortening the effective stripping path. For this reason, the $\mathrm{H}^{0}$ horizontal emittance $\varepsilon_{x}$ calculated with realistic fields is expected never to exceed the value calculated with constant field.

\section{Bending magnet}

After the magnetic neutralizer, a conventional bending dipole magnet is required to separate $\mathrm{H}^{-}$from $\mathrm{H}^{0}$. To limit the Lorentz stripping of $\mathrm{H}^{-}$below $1 \mathrm{~W} / \mathrm{m}$, the magnetic field is kept at $0.3 \mathrm{~T}$. The dipole aperture is $100 \mathrm{~mm}$ and its radius is $18.86 \mathrm{~m}$. Its length is $2 \mathrm{~m}$, giving sufficient separation between the two beam lines (about $10.5 \mathrm{~cm}$ ) to insert a stripping section at the dipole exit.

\section{Stripper foil}

A carbon foil stripper is placed across the $\mathrm{H}^{0}$ path to turn the neutral beam into a proton beam. In our case, the stripper is inserted $10 \mathrm{~cm}$ after the bending magnet. A suitable space is required for the foil and for the horizontal foil changer. A cooled electron collector [13] is foreseen, to collect the electrons produced by the foil. 

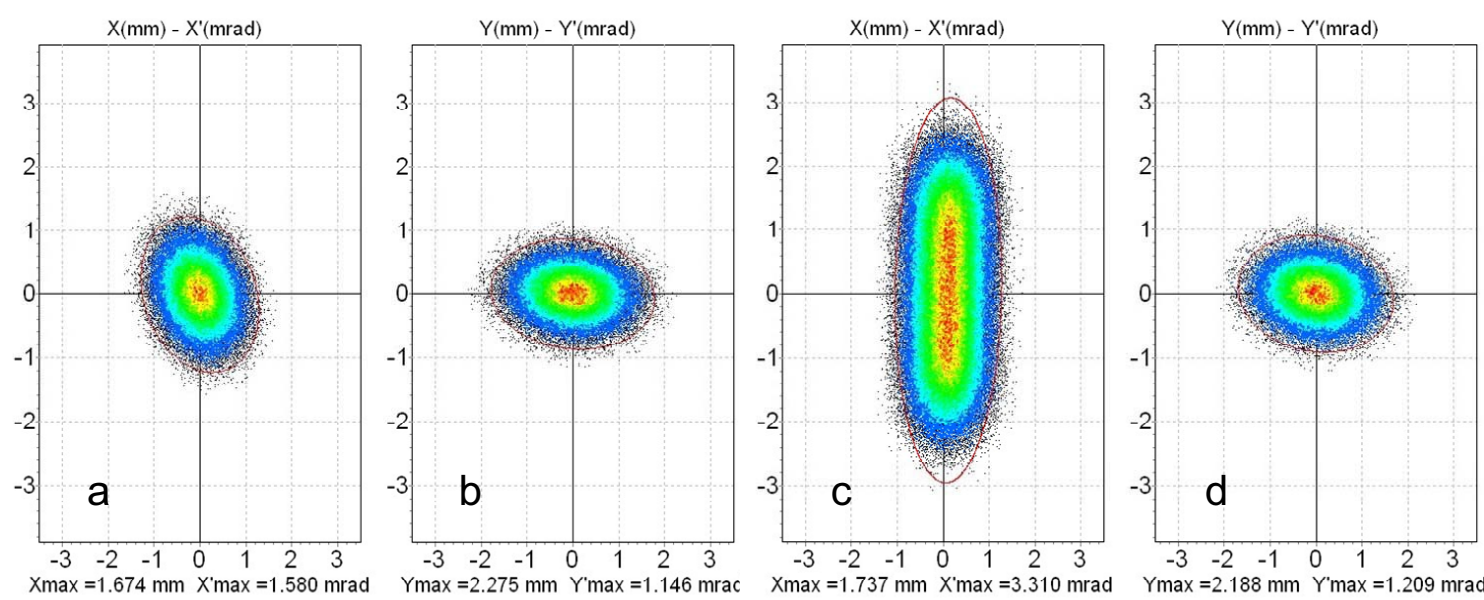

FIG. 4. (Color) Horizontal and vertical phase spaces of the main $\mathrm{H}^{-}$beam at the entrance (left), and of the $\mathrm{H}^{0}$ beam at the exit (right) of the second wiggler magnet.

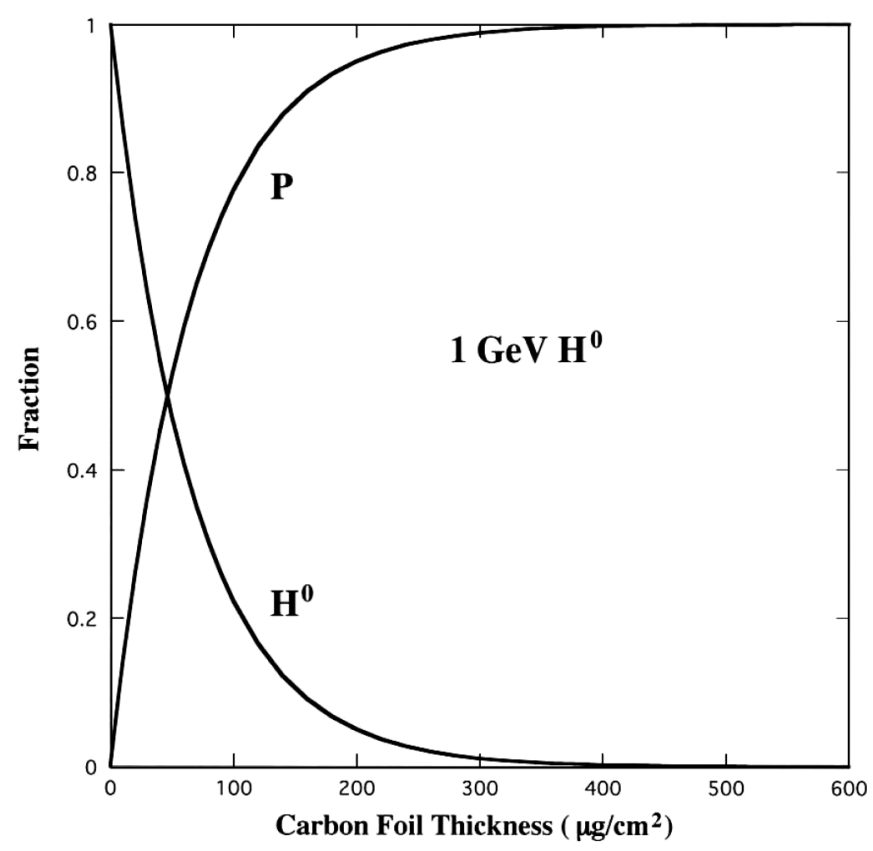

FIG. 5. $\quad \mathrm{P}$ and $\mathrm{H}^{0}$ fraction versus carbon foil thickness, for a $1 \mathrm{GeV} \mathrm{H}^{0}$ primary beam. The $\mathrm{H}^{-}$fraction is negligible.
For $1 \mathrm{GeV}, \mathrm{H}^{0}$ beam, the stripping efficiency (Fig. 5) can be calculated using the following cross sections [14]:

$$
\sigma_{01}=3.0 \times 10^{-19} \mathrm{~cm}^{2} .
$$

At this energy, a carbon foil thickness of $500 \mu \mathrm{g} / \mathrm{cm}^{2}$ converts $\sim 99.95 \%$ of all the $\mathrm{H}^{0}$ particles to protons, resulting in only $\sim 50 \mathrm{~W}$ of unconverted $\mathrm{H}^{0}$ beam. A part of these $\mathrm{H}^{0}$ atoms, however, leave the foil in an excited state with $n>2$ and may be stripped in the subsequent magnets, creating a halo and possible beam losses $[9,15,16]$. The current of this part is roughly 1 order of magnitude lower than the total $\mathrm{H}^{0}$ one. In our case, these losses are not expected to exceed a few watts. Moreover, since the cross section for electron pickup is very small for energies above $100 \mathrm{keV}$ [17], the $\mathrm{H}^{-}$fraction is negligible.

The lifetime of the stripping carbon foils primarily depends on three factors: beam current density, foil thickness, and foil preparation method. The estimated heat load in the foil is $\sim 0.1 \mathrm{~W}$; similar foils, in similar beam conditions, have shown lifetimes of several weeks [18]. In addition to foils such as the hybrid boron mixed carbon foil [19] and the diamond foil [18], the feasibility of the carbon nanotube foil [20] is also studied.

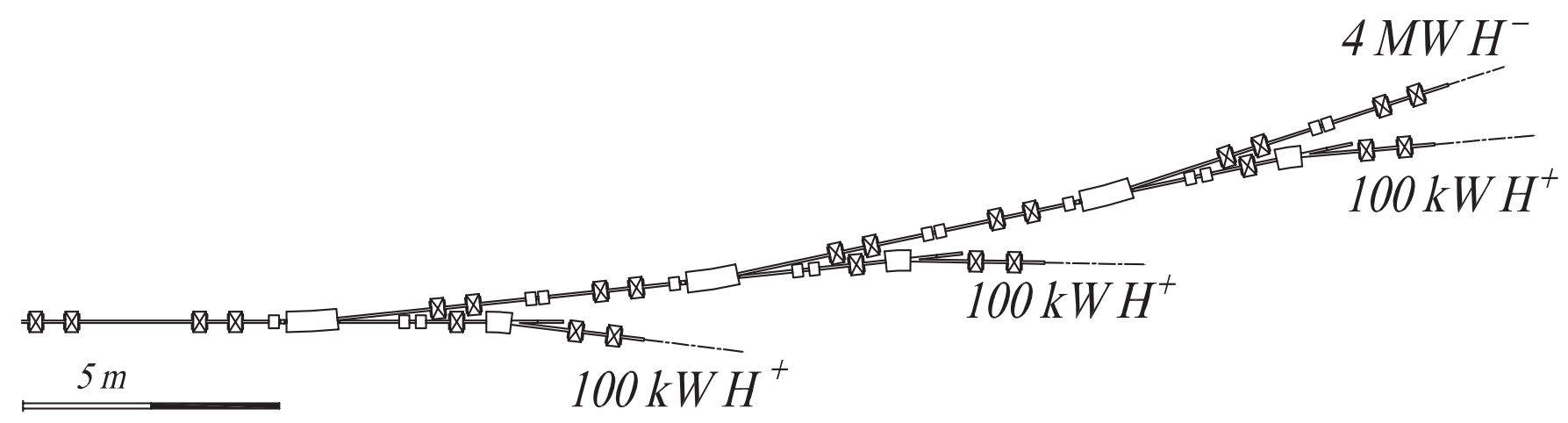

FIG. 6. Schematic layout of the extraction area and the three beam splitters. 

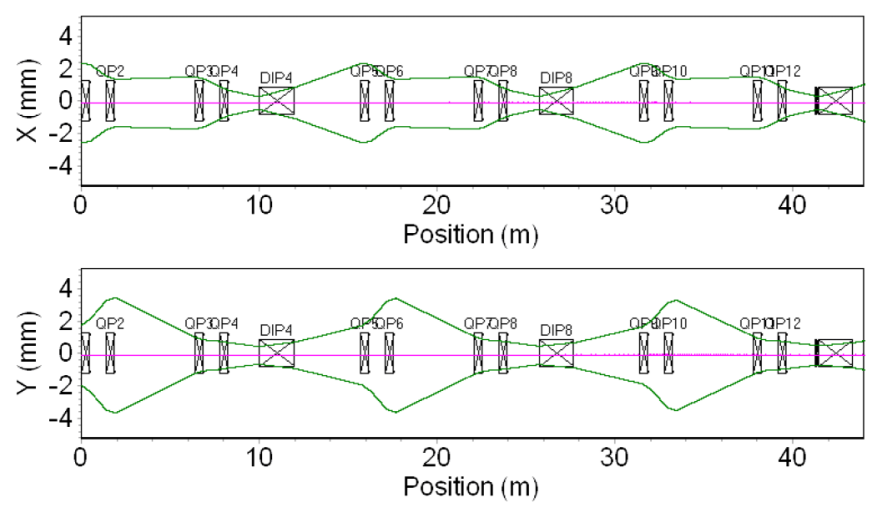

FIG. 7. (Color) $\mathrm{H}^{-}$beam rms envelope along the three splitting sections, calculated with the TRACEWIN-PARTRAN codes.

After stripping, the proton beam is further bent by a dipole magnet, similar to the previous one, and finally extracted. The weak current of the unbent neutral atoms is mainly collected by a beam dump (BD in Fig. 1), while the excited $\mathrm{H}^{0}$ which are stripped in the dipole are lost in the vacuum chamber.

Negligible emittance growth induced by the foil is expected, thus a second foil can be placed along the line, a few centimeters apart. This significantly reduces the probability of transmitting the full $100 \mathrm{~kW}$ to the $\mathrm{H}^{0}$ beam dump in case of rupture of one foil.

\section{6. $\mathrm{H}^{-}$beam transport}

To spill three parallel high power proton beams from the main $\mathrm{H}^{-}$beam, three identical splitting sections are used in series (Fig. 6).

At the wigglers, the beam aperture is necessarily narrow $(30 \mathrm{~mm})$, and a waist in both horizontal and vertical planes is required, at those locations, to prevent losses. The system is designed in order to reproduce the same beam transport in each splitting section (Fig. 7). No transverse emittance growth is observed, while the longitudinal emittance is increased by $\sim 3 \%$ after each splitter. This has no serious consequences in this particular application; in case of a larger number of splitting stations, however, an achromatic design should be considered.

\section{7. $\mathrm{H}^{0}$ and $\mathrm{H}^{+}$beam transport}

By properly choosing the Twiss parameters at the wiggler, the neutral beam can be made to travel to the stripper with an acceptable increase in the transverse size.

After the stripper, the so-generated protons are focused and separated from the weak residual neutral current by a second dipole magnet, and finally transported to the RIB source target. The proton beam emittance and size are always well suited for a low loss transport along standard, $100 \mathrm{~mm}$ diameter beam pipes.

\section{CONCLUSIONS}

The high power, $\mathrm{cw} \mathrm{H}^{-} / \mathrm{H}^{+}$beam splitter system described above, based on a magnetic neutralizer and a carbon foil stripper, is feasible with realistic parameters. The extracted beam current of the secondary lines is finely adjustable without perturbing the main $\mathrm{H}^{-}$beam. The $500 \mu \mathrm{g} / \mathrm{cm}^{2}$ carbon foil stripper is expected to receive a modest heat load, leading to foil lifetime of the order of several weeks. The emittance growth of the primary $\mathrm{H}^{-}$ beam through the splitter is negligible, and the splitting process can be repeated many times. The emittance of the secondary $\mathrm{H}^{0}$ beam after the magnetic neutralizer (less than a factor of 3 from the primary beam value) is fully acceptable for the EURISOL scope of bombarding a RIB source target.

The EURISOL scheme might be used for different applications, with different beam power values in the extraction lines, the limit being only the stripper foil lifetime. As an example, the beam might be completely neutralized and sent to the $\mathrm{H}^{+}$lines without the necessity of transporting it to the $\mathrm{H}^{-}$target (e.g. during maintenance time). The same method might be used for pulsed beams as well, allowing in some cases elimination of choppers and kickers.

\section{ACKNOWLEDGMENTS}

We are grateful to D. Zenere for his help in the splitter layout design. We thank R. Duperrier, D. Uriot, A. Balabin, and $\mathrm{M}$. Comunian for their precious suggestions in the use of the TRACEWIN-PARTRAN set of codes. We acknowledge the financial support of the European Community under the FP6 "Research Infrastructure Action-Structuring the European Research Area" EURISOL DS Project Contract No. 515768 RIDS.

[1] http://www.eurisol.org/site01/index.php.

[2] J. Alonso, The 7th ICFA Mini-Workshop on High Intensity, High Brightness Hadron Beams, Lake Como, Winsconsin, 1999, edited by N. V. Mokhov and W. Chou, Vol. 51, http://lss.fnal.gov/archive/2000/conf/Conf-00185.pdf.

[3] R. Hutson, D. Fitzgerald, S. Frankle, R. Macek, M. Plum, and C. Wilkinson, Proceedings of Particle Accelerator Conference, Washington DC, USA, 1993, Vol. 366.

[4] Isao Yamane, Phys. Rev. ST Accel. Beams 1, 053501 (1998).

[5] V. Danilov, A. Aleksandrov, S. Assadi, S. Henderson, N. Holtkamp, T. Shea, A. Shishlo, Y. Braiman, Y. Liu, J. Barhen, and T. Zacharia, Phys. Rev. ST Accel. Beams 6, 053501 (2003).

[6] V. Danilov, A. Aleksandrov, S. Assadi, J. Barhen, W. Blokland, Y. Braiman, D. Brown, C. Deibele, W. Grice, S. Henderson, J. Holmes, Y. Liu, A. Shishlo, A. Webster, and I. N. Nesterenko, Phys. Rev. ST Accel. Beams 10, 053501 (2007).

[7] Leonard R. Scherk, Can. J. Phys. 57, 558 (1979). 
[8] A. J. Jason, D. W. Hudgings, and O. B. Van Dyck, IEEE Trans. Nucl. Sci. NS-28, 2704 (1981).

[9] R. Hutson, Proceedings of the Particle Accelerator Conference, San Francisco, CA, USA, 1991, Vol. 955.

[10] R. Duperrier, N. Pichoff, and D. Uriot, Proceedings of the ICCS-International Conference on Computational Science, Amsterdam, 2002, Part III.

[11] David C. Carey, The Optics of Charged Particle Beams (Harwood Academic Publishers, Chur, Switzerland, 1987), Chap. 5.3, p. 84.

[12] K. L. Brown, SLAC Report No 75, 1982, http://www.slac. stanford.edu/pubs/slacreports/reports01/slac-r-075.pdf.

[13] Y. Lee, G. Mahler, W. Meng, D. Raparia, L. Wang, and J. Wei, Proceedings of the Particle Accelerator Conference, Knoxville, USA, 2005, Vol. 2384.

[14] R. C. Webber and C. Hojvat, IEEE Trans. Nucl. Sci. NS26, 4012 (1979).

[15] J. Donahue, D. Clark, S. Cohen, D. Fitzgerald, S. Frankle, R. Hutson, R. Macek, E. Mackerrow, O. van Dyck, C.
Wilkinson, H. Bryant, M. Gulley, M. Halka, P. Keating, and W. Miller, Proceedings of the Particle Accelerator Conference, Washington, DC, USA, 1993, Vol. 369.

[16] M.S. Gulley, P. B. Keating, H. C. Bryant, E.P. MacKerrow, W. A. Miller, D.C. Rislove, Stanley Cohen, J. B. Donahue, D. H. Fitzgerald, S. C. Frankle, David J. Funk, R. L. Hutson, R. J. Macek, M. A. Plum, N. G. Stanciu, O. B. van Dyck, C. A. Wilkinson, and C.W. Planner, Phys. Rev. A 53, 3201 (1996).

[17] H. Tawara and A. Russek, Rev. Mod. Phys. 45, 178 (1973).

[18] T. Spickermann, J. Borden, R. J. Macek, R. W. Shaw, C. S. Feigerle, and I. Sugai, Proceedings of ICFA-HB2006, Tsukuba, Japan, 2006, Vol. 116.

[19] I. Sugai, Y. Takeda, M. Oyaizu, H. Kawakami, Y. Irie, K. Hara, A. Takagi, H. Hattori, K. Kawasaki, J. Kamiya, and M. Kinsho, Nucl. Instrum. Methods Phys. Res., Sect. A 561, 16 (2006).

[20] T. Saito (private communication). 